\title{
Video Article \\ Optimization of a Multiplex RNA-based Expression Assay Using Breast Cancer Archival Material
}

\author{
Shawn Baldacchino ${ }^{1}$, Christian Saliba ${ }^{2}$, Jeanesse Scerri ${ }^{3}$, Christian Scerri ${ }^{3}$, Godfrey Grech \\ ${ }^{1}$ Department of Pathology, Faculty of Medicine \& Surgery, University of Malta \\ ${ }^{2}$ Centre for Molecular Medicine and Biobanking, University of Malta \\ ${ }^{3}$ Department of Physiology \& Biochemistry, Faculty of Medicine and Surgery, University of Malta
}

Correspondence to: Godfrey Grech at godfrey.grech@um.edu.mt

URL: https://www.jove.com/video/57148

DOI: doi: $10.3791 / 57148$

Keywords: Bioengineering, Issue 138, Biomarkers, translational medicine, breast cancer, FFPE, diagnosis, classification, cancer subtypes, HER2, estrogen receptor, triple negative, multiplex bead-based assay, XMAP

Date Published: 8/1/2018

Citation: Baldacchino, S., Saliba, C., Scerri, J., Scerri, C., Grech, G. Optimization of a Multiplex RNA-based Expression Assay Using Breast Cancer Archival Material. J. Vis. Exp. (138), e57148, doi:10.3791/57148 (2018).

\section{Abstract}

Nucleic acid degradation in archival tissue, tumor heterogeneity, and a lack of fresh frozen tissue specimens can negatively impact cancer diagnostic services in pathology laboratories worldwide. Gene amplification and expression diagnostic testing using archival material or material that requires transportation to servicing laboratories, needs a more robust and accurate test adapted to current clinical workflows. Our research team optimized the use of Invitrogen ${ }^{\mathrm{TM}}$ QuantiGene ${ }^{\mathrm{TM}}$ Plex Assay (Thermo Fisher Scientific) to quantify RNA in archival material using branchedDNA (bDNA) technology on Luminex $x M A P^{\circledR}$ magnetic beads. The gene expression assay described in this manuscript is a novel, quick, and multiplex method that can accurately classify breast cancer into the different molecular subtypes, omitting the subjectivity of interpretation inherent in imaging techniques. In addition, due to the low input of material required, heterogeneous tumors can be laser microdissected using Hematoxylin and Eosin (H\&E) stained sections. This method has a wide range of possible applications including tumor classification with diagnostic potential and measurement of biomarkers in liquid biopsies, which would allow better patient management and disease monitoring. In addition, the quantitative measurement of biomarkers in archival material is useful in oncology research with access to libraries of clinicallyannotated material, in which retrospective studies can validate potential biomarkers and their clinical outcome correlation.

\section{Video Link}

The video component of this article can be found at https://www.jove.com/video/57148/

\section{Introduction}

Optimization of RNA based assays using archival formalin-fixed paraffin-embedded (FFPE) material is challenging due to variability in surgical tissue processing and degradation of RNA caused by formalin used for tissue integrity preservation ${ }^{1,2}$. To overcome the limitation of performing accurate gene expression studies from archival material, our group used the bDNA multiplex magnetic bead assay. Instead of enzymatic amplification of a target template, the bDNA technology uses hybridization of specific probes and amplification of a reporter signal ${ }^{3}$. The short recognition sequences of the capture and detection probes are designed to hybridize to short fragments of target RNA ${ }^{4}$. In addition, the use of tissue homogenates as the direct starting material in this assay, overcomes the inevitable loss of RNA that results from assays requiring prior RNA extraction and purification. Signal amplification, the use of short recognition sequences, and the exclusion of a purification step, contribute to the reduce in technical variation of the assay. The technology provides the possibility to multiplex the assay (up to 80 RNA targets) and measure the expression of a panel of targets from low material input. This protocol describes the preparation and staining of tissue samples for laser microdissection. Staining on the membrane slides facilitate the imaging of the tumor and histological architecture to provide accurate selection and profiling of: (1) the tumor and normal ducts in breast tissue, and (2) the malignant cell clones within heterogeneous tumors.

Molecular classification of breast cancer is a process that interrogates molecular markers to categorize patient tumors into three molecular classes, i.e., luminal, human epidermal growth factor receptor 2 (HER2)-enriched, and basal subtype. The HER2-enriched subtype is well defined, with high expression of HER2 receptor, due to the ERBB2 gene amplification, combined with low or absent estrogen receptor (ER) and progesterone receptor $(\mathrm{PgR})$. The luminal subtype is generally positive for ER and the basal subtype are in general negative for the three receptors (HER2, ER, PgR), and significantly overlaps with the triple negative breast cancer (TNBC) diagnostic subtype ${ }^{5,6}$. Other markers are used to determine epithelial and mesenchymal characteristics. Fibronectin (FN1) is a main component of the breast tissue mesenchymal compartment. Increased FN1 expression is accompanied by high Ki67 staining, and shows a signature for a more invasive tumor ${ }^{7,8}$ and is associated with metastasis ${ }^{9}$. Interestingly, FN1 was found to be present in microvesicles originating from the tumor cells, that induced activation of mitogenic signals in recipient fibroblasts ${ }^{10}$. Hence, circulating microvesicles such as exosomes are a potential marker of early detection or metastasis and relapse ${ }^{11}$

Tumor area selection for breast cancer transcriptional subtyping has recurrently been performed by macrodissection ${ }^{12,13}$. To overcome tissue heterogeneity and increase sensitivity, we have reliably combined classical tissue staining with multiplex molecular profiling methods. As a 
proof of principle, two distinct breast cancer clones have been defined by their epithelial mesenchymal signature and metastatic potential. The workflow of the described protocol can be easily translated to the current clinical setup and used to selectively isolate and characterize tissue subtypes using targeted mRNA profiling.

\section{Protocol}

Ethical approval for use of breast tissue material in this study was obtained from the University Research Ethics Committee (UREC) of the University of Malta (Ref: 22/2012).

\section{Tissue Preparation}

1. Using appropriate $H \& E$ procedures ${ }^{14}$, prepare stained reference tissue sections and digitally scan the slides for reference during microdissection.

2. Using a microtome, cut $20 \mu \mathrm{m}$ tissues sections onto an RNAse-free water bath at $40{ }^{\circ} \mathrm{C}$. Collect the sections on membrane slides for laser microdissection using clean, RNase-decontaminated equipment and materials.

3. Dry the membrane slides at $37^{\circ} \mathrm{C}$ in a drying incubator overnight. Stain using the appropriate H\&E procedure ${ }^{15}$ with molecular biology grade solutions and increasing the staining time of Hematoxylin to 6 min. If required, stain the membrane slides with the appropriate immunohistochemical protocols ${ }^{16}$.

\section{Laser Microdissection}

1. Set the slide limits and calibrate the stage movement.

1. Move the laser view onto an empty area of a membrane slide. Calibrate the laser focus by firing a laser shot at increasing focus intervals $(5 \%)$ until the laser starts to pierce through the membrane. Draw and microdissect any shape and finetune the laser focus to maximize the laser efficiency during the laser dissection.

2. Increase the stage movement speed to a point where the laser dissection efficiency is not compromised. Calibrate the laser at the selected objective lens and re-calibrate if changing objectives (laser speed at $1-15 \%$, focus at $70-75 \%$, and power at $90-100 \%$ when using the $4 \mathrm{X}$ objective).

2. Scan the stained membrane slides using the $4 X$ objective.

3. Select and encircle the target areas for microdissection on the membrane slide. Laser dissect a minimum area of $42 \mathrm{~mm}^{2}$. Record the area dissected to determine the volume of sample homogenizing buffer to be used.

4. Use the cap lift mechanism to retrieve the dissected section onto the diffuser caps of labeled tubes attached to the appropriate appendage. If the dissected section is not retrieved onto the cap, repeat the laser dissection of the area.

\section{Tissue Lysis}

Note: Several solutions and materials are supplied along with kits mentioned in the Table of Materials.

1. Prepare the required homogenizing mixture volume for sample lysis using homogenizing solution and proteinase $\mathrm{K}$ at a $60: 1$ ratio.

2. Pipette $2.4 \mu \mathrm{L}$ of homogenizing solution into each tube for each $1 \mathrm{~mm}^{2}$ of tissue area, vortex for $10 \mathrm{~s}$ at the maximum speed, and centrifuge at room temperature for $5 \mathrm{~s}$ at $2,500 \times \mathrm{g}$.

3. Incubate in a heating block at $65^{\circ} \mathrm{C}$ for $12-18 \mathrm{~h}$ with shaking at $600 \mathrm{rpm}$.

4. Centrifuge the lysates at $21,000 \times \mathrm{g}$ for $5 \mathrm{~min}$ at room temperature.

5. Using a pipette, carefully aspirate the clear supernatant and dispense into a labeled fresh tube. Avoid aspirating the tissue/membrane fragments as this may reduce the quality of results.

6. Store the clear supernatant in a $-80^{\circ} \mathrm{C}$ freezer.

\section{Hybridization-based Assay}

1. Perform the following preparations.

1. Pre-warm the lysis mixture by placing the reagent bottle in an incubator at $37^{\circ} \mathrm{C}$ for 30 min and mix gently by inversion.

2. If the tissue homogenates are frozen, thaw at room temperature and incubate at $37^{\circ} \mathrm{C}$ for 30 min. Vortex the samples at the maximum speed following the incubation.

3. Set the shaking incubator at $54{ }^{\circ} \mathrm{C}$ and place the probe of the temperature validation kit in an empty well of a 96-well plate. After $2 \mathrm{~h}$ check the temperature on the digital thermometer and set the delta temperature of shaking incubator to correct for any temperature difference.

4. Thaw and vortex the probe set and blocking reagent for $10 \mathrm{~s}$, centrifuge at room temperature for $5 \mathrm{~s}$ at $2,500 \times \mathrm{g}$, and keep on ice.

5. Keep the Proteinase $\mathrm{K}$ vial on ice during use.

6. Sonicate the capture beads at $46 \mathrm{KHz}$ for $3 \mathrm{~min}$, then vortex at the maximum speed for another $3 \mathrm{~min}$.

2. Prepare a $25 \mu \mathrm{L}$ working bead mix per well by scaling up the following reagents accordingly: $4.25 \mu \mathrm{L}$ of Nuclease-free water, $16.65 \mu \mathrm{L}$ of lysis mixture, $1.00 \mu \mathrm{L}$ of blocking reagent, $0.10 \mu \mathrm{L}$ of Proteinase $\mathrm{K}, 0.50 \mu \mathrm{L}$ of capture beads, $2.50 \mu \mathrm{L}$ of probe set.

3. Vortex the working bead mix for $10 \mathrm{~s}$ at maximum speed, then pipette $25 \mu \mathrm{L} /$ well into the magnetic separation plate.

4. Pipette $25 \mu \mathrm{L}$ tissue homogenate to a magnetic separation 96-well plate and $25 \mu \mathrm{L}$ homogenizing solution to 3 wells as the blanks.

5. Seal the magnetic separation plate using a clear plastic pressure seal and place the plate in the incubator at $54 \pm 1^{\circ} \mathrm{C}$ for $18-22 \mathrm{~h}$ while shaking at $600 \mathrm{rpm}$. 
6. Prepare a $1 \mathrm{X}$ wash buffer solution for 24 wells by mixing $31.5 \mathrm{~mL}$ of RNase-free water with $0.1 \mathrm{~mL}$ of wash buffer component $1 \mathrm{and} 1.67 \mathrm{~mL}$ of wash buffer component 2.

7. Remove the plate from incubator. Set the temperature of the shaking incubator to $50 \pm 1{ }^{\circ} \mathrm{C}$.

8. Mount the magnetic separation plate on the hand-held magnetic plate washer and lock in place. Remove the pressure seal and wait 1 min for the magnetic beads to settle.

9. Washing Step: Invert the magnetic separation plate mounted onto the plate over the sink and blot gently on a tissue paper to remove residual solution. Using a multichannel pipette add $100 \mu \mathrm{L}$ of $1 \mathrm{X}$ wash buffer solution into each well and wait $15 \mathrm{~s}$. Repeat this step to wash the plate 3 times.

10. Pipette $50 \mu \mathrm{L}$ of the pre-amplifier reagent to each well. Seal the plate with an aluminum plate seal and shake the plate at $800 \mathrm{rpm}$ for $1 \mathrm{~min}$ at room temperature. Incubate for $1 \mathrm{~h}$ at $50 \pm 1{ }^{\circ} \mathrm{C}$ while shaking at $600 \mathrm{rpm}$.

11. Repeat step 4.9 (Washing step).

12. Pipette $50 \mu \mathrm{L}$ of the amplifier reagent to each well. Seal the plate with an aluminum plate seal and shake the plate as in step 4.10 .

13. Repeat step 4.9 (Washing step).

14. Vortex the label probe for $10 \mathrm{~s}$ at maximum speed. Add $50 \mu \mathrm{L}$ of the label probe to each well. Seal and shake the plate (step 4.10).

15. Repeat Step 4.9 (Washing step).

16. Vortex the Streptavidin phycoerythrin (SAPE) for $10 \mathrm{~s}$ at maximum speed. Add $50 \mu \mathrm{L}$ of SAPE to each well. Seal and shake the plate (step 4.10).

17. Repeat Step 4.9 (Washing step) except use the SAPE wash buffer instead of the wash buffer solution.

18. Add $130 \mu \mathrm{L}$ of SAPE wash buffer to each well and seal plate with an aluminum plate seal. Shake the plate at $800 \mathrm{rpm}$ at room temperature for $3 \mathrm{~min}$.

19. Start-up the magnetic bead analyzer. Perform the calibration and verification routine by following the manufacturer's instructions.

20. Set up a protocol on the magnetic bead analyzer using the following parameters: Sample Size: $100 \mu \mathrm{L} ; \mathrm{DD}$ Gate: 5,000-25,000; Timeout: 90 s; Bead Event/Bead Region: 100; and click next. Select the respective bead numbers in the panel and define the bead regions with the respective target genes as indicated by the manufacturer.

21. Add a new analysis by selecting "Create Batch using an existing protocol" from the Batches tab. In the plate layout designate the wells as unknown or blank, respectively and provide a label for each sample in the Samples Panel.

22. Remove the aluminum plate seal from the 96-well reaction plate. Eject the plate tray by clicking on the Eject button, load the plate into the magnetic bead analyzer. Click Retract| Run Batch to initiate the analyzer. After reading, eject and remove the 96-well plate. Clean and shut down the magnetic bead analyzer as recommended by the manufacturer.

\section{Data Analysis}

1. In the Batch| Results tab, select the respective analysis file and click the export to.csv button. Open the.csv file using a spreadsheet software.

2. Observe the bead counts and omit any results with $<40$ beads/region.

3. Average out the values of the blank wells and work out the standard deviation (SD) and Limit of Detection (LOD) (blank average $+(3 \times S D)$ ) per target gene. Set the values lower than the LOD as undetected.

Note: If any of the normalizing expression values are undetected, regard the sample data as inadequate. Consider expression values over 20,000 as over the upper sensitivity limit. The lysates of these samples should be diluted and re-analyzed.

4. Subtract the blank average from the raw data per gene.

5. Compute the geometric mean of the selected normalizing genes per sample. Normalize individual sample data to the respective geometric mean.

6. Analyze the data on data science platforms or using defined algorithms.

1. Import the data into the data science platform by clicking Add Data. Drag the data file into the Process Workspace and connect to a [Select Attributes] operator, then to a Principal component analysis (PCA) operator, and then to the result port.

2. In the Select Attribute operator select the subset of normalized gene data and a nominal variable such as breast cancer subtype. Run the process by clicking Play. In the Charts Tab select the "Scatter 3D Color" in the Chart style and the nominal variable in the Color field. Assess the PCA data variability on a 3-dimensional plot.

\section{Representative Results}

The described method has been applied for the simultaneous measurement of 40 transcripts in H\&E stained (Figure 1), microdissected (Figure 2) highly degraded FFPE material. Using this method, we show the accurate characterization of receptor status (Figure 3A), classification of tumors into luminal and basal molecular subtypes ${ }^{17}$, and differential expression of the mesenchymal marker, FN1, when comparing tumor and matched control tissue (Figure 3B), in the various receptor positive and negative subtypes. 


\section{Correlation between stained and unstained tissue}

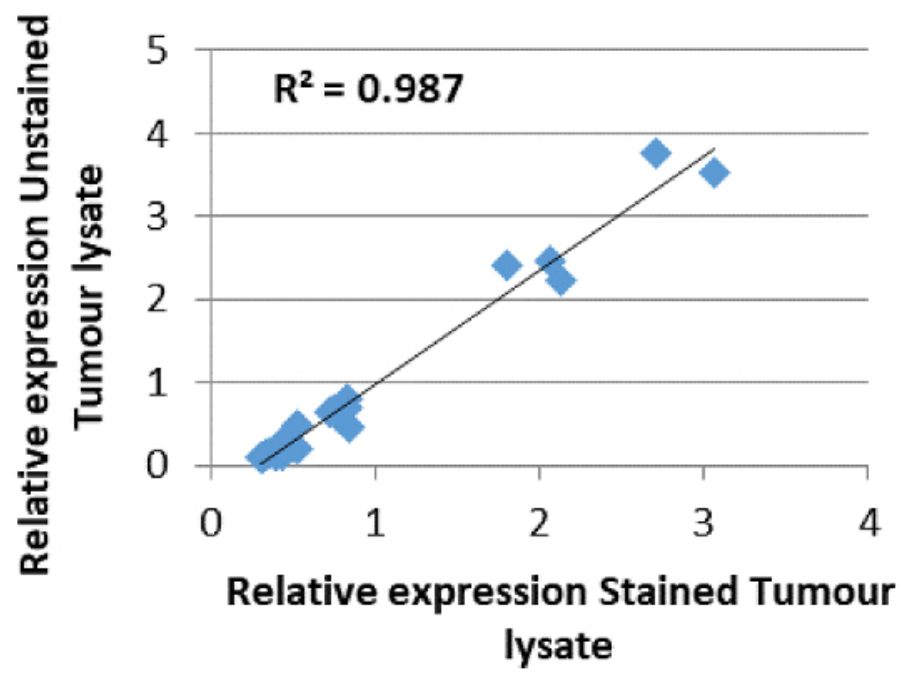

Figure 1: Gene expression using H\&E stained material. Correlation between an expression profile derived from an unstained tumor section as compared to a stained tumor section. [Pearson Correlation $p$-value $=5.34 \mathrm{E}-26$ ]. Reproduced with permission ${ }^{17}$. Please click here to view a larger version of this figure.
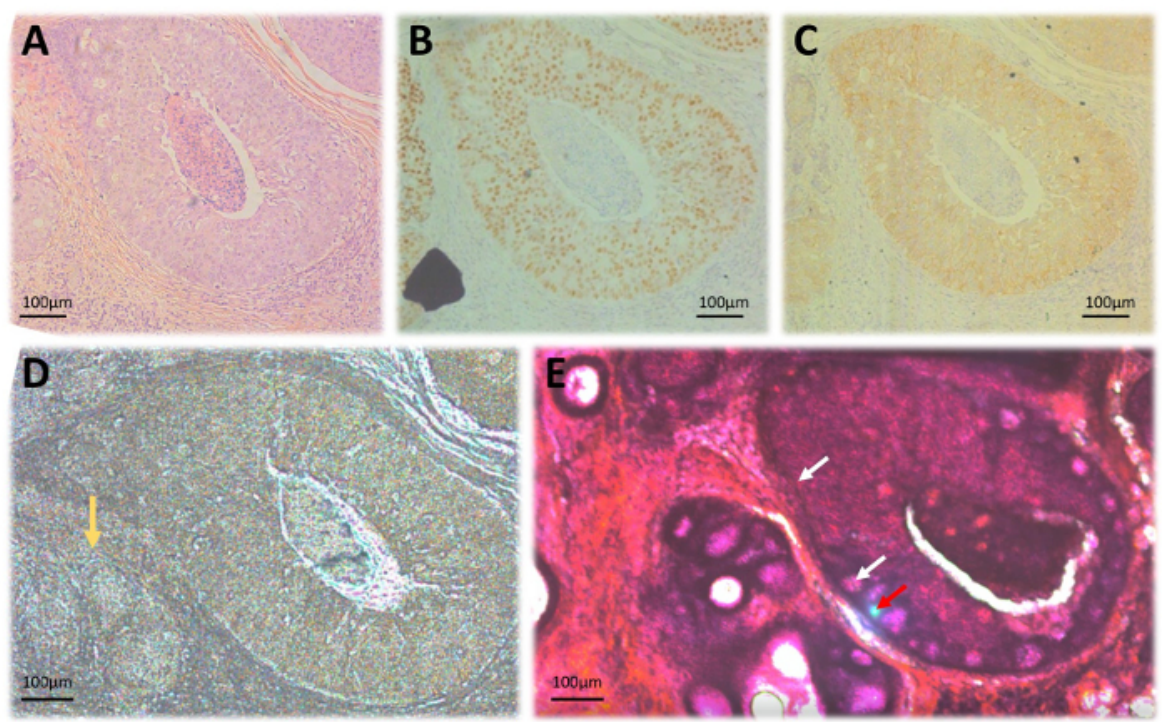

Figure 2: Laser microdissection of FFPE tissues. (A) H\&E stained slide; (B) Immunohistochemical staining for ER expression; (C) HER2 immunohistochemical staining; (D) Unstained $20 \mu \mathrm{m}$ section on laser microdissection membrane slides. The yellow arrow indicates an area of invasive tumor that is not clearly demarcated due to lack of staining. (E) A $20 \mu \mathrm{m}$ section stained with H\&E for better delineation of areas of interest. White arrows indicate laser dissection trail while the Red arrow shows the laser focus during dissection. All illustrations were captured at 10x magnification. Please click here to view a larger version of this figure. 


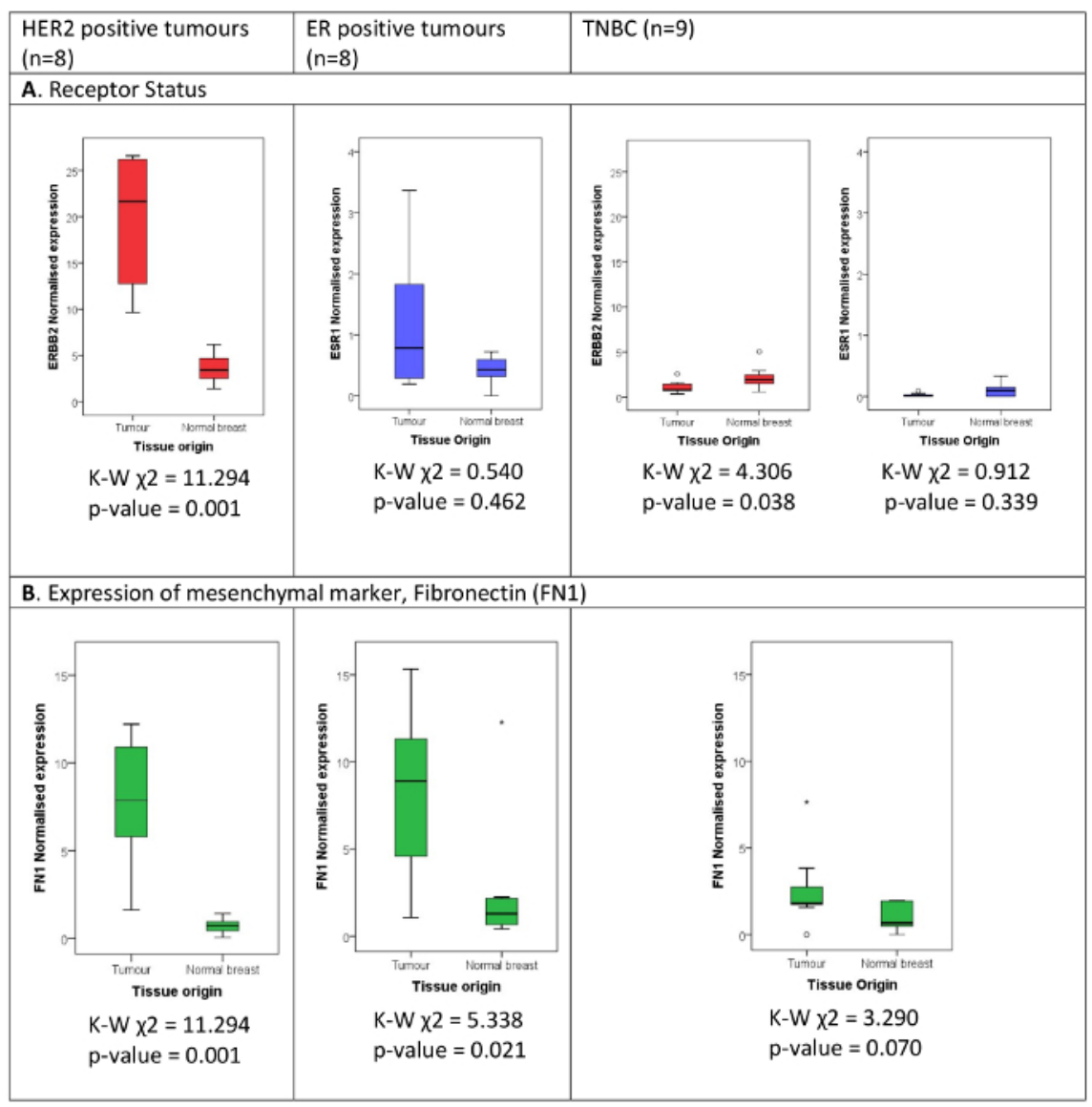

Figure 3: Expression of (A) receptor status and (B) mesenchymal marker FN1, in breast tumor compared to matched normal.

The classical diagnostic breast cancer subtypes are defined as per diagnostic result using immunohistochemistry and fluorescence in situ hybridization (FISH) for HER2 equivocal immunohistochemical staining. The normalized expression of (A) HER2 and ER, (B) FN1 measured by the hybridization-based assay is illustrated in HER2 positive, ER positive, and TNBC cases compared to patient matched normal breast tissue. The Kruskal Wallis Test Statistic $\left(\mathrm{K}-\mathrm{W} \mathrm{X}^{2}\right)$ shows that the expression of HER2 is significantly $(p<0.05)$ higher in the tumor tissue as opposed to the matched normal tissue in the HER2 positive cohort and significantly lower in the TNBC cohort. ER expression was not found to be significantly higher in the tumor tissue as opposed to the matched normal in the ER positive and TNBC cohorts. FN1 is significantly higher in the tumor tissue in the HER2 and ER positive subtypes and shows a trend towards being significantly elevated in tumor tissue also in the TNBC cohort. Please click here to view a larger version of this figure. 


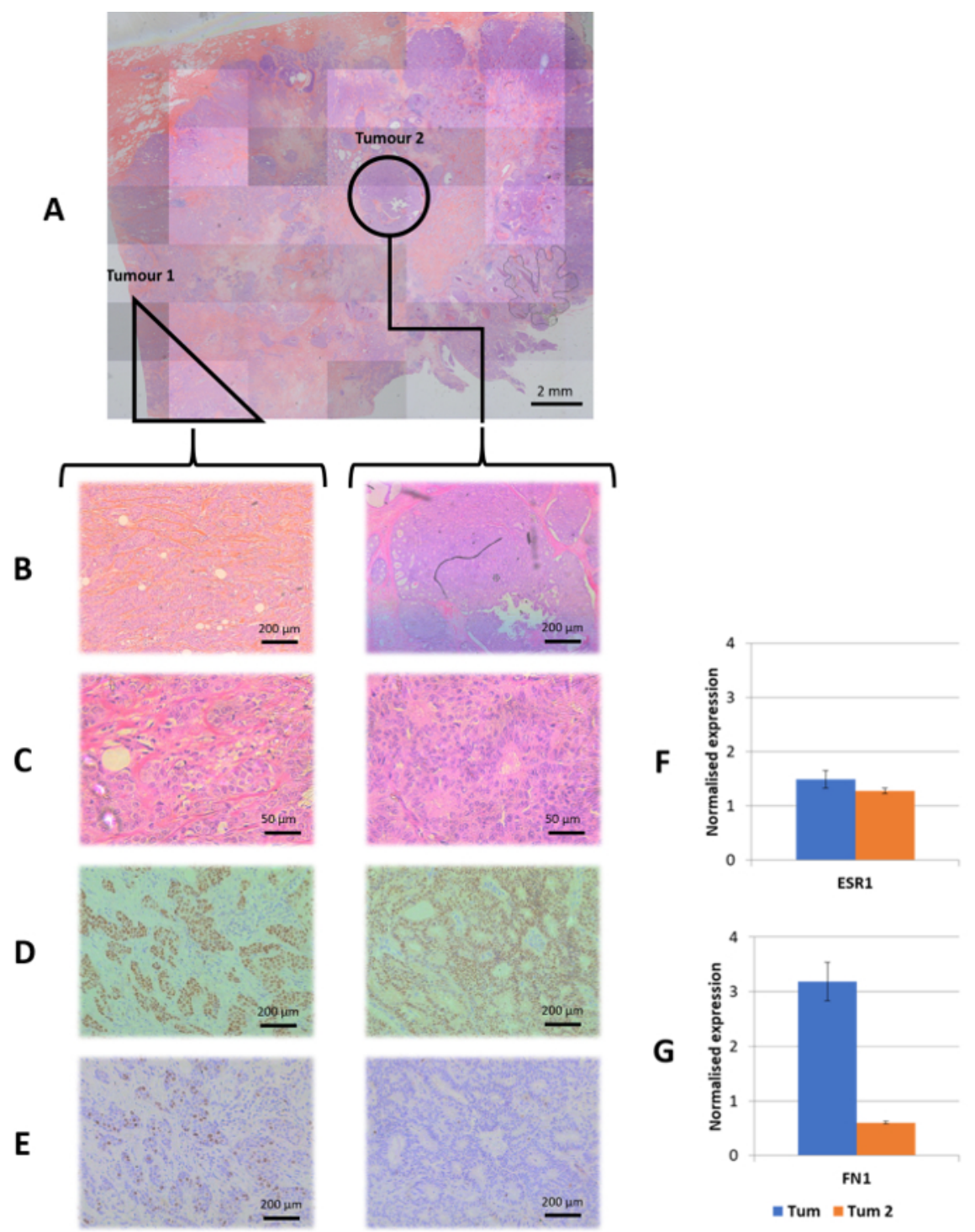

Figure 4: Case study: tumor heterogeneity. Morphologically distinct tumors were microdissected and treated as distinct samples. (A) The master scan of the H\&E section. (B, C) A 10x and 40x magnification, respectively for each tumor morphology identified. (D) Immunohistochemical staining for ER at 10x magnification. (E) Immunohistochemical staining for Ki67 at 10x magnification showing a higher mitotic activity in tumor 1. (F) Normalized expression levels for the ESR1 gene in each tumor showing relatively high and equal expression between tumors as expected from the immunohistochemical result. (G) Normalized expression levels of FN1, a mesenchymal marker, where increased FN1 expression is accompanied by high Ki67 staining showing a signature for a more invasive tumor. The inverse is observed in tumor 2, which appears to be a slower proliferating tumor with a lower malignant potential represented by reduced FN1 expression. Please click here to view a larger version of this figure.

\section{Discussion}

A bead-based multiplex bDNA assay was optimized to quantify gene expression on degraded RNA derived from FFPE breast cancer tissue and normal breast ducts. Optimizing the assay, involved developing an algorithm to classify breast cancer tumors in luminal and basal subtypes utilizing 8 well-known biomarkers and 5 potential normalizing genes. Data normalization was done using permutations of the normalizing genes. The selection of the normalizing genes was based on the best prediction of receptor status using the Luminal/Basal classifier genes. To classify Luminal/Basal subtypes from FFPE tissues, the normalizing genes selected were Beta-actin (ACTB), Glyceraldehyde 3-phosphate dehydrogenase (GAPDH), and Hypoxanthine Phosphoribosyltransferase 1 (HPRT1).

The method can be adapted for use in other diagnostic and research areas following adequate selection of the normalizing gene set. One important application of this method in the research sector is the measurement of biomarkers in archival material that is well annotated with clinical outcomes. This could validate potential predictive markers in retrospective studies, quickly and accurately, and avoid long-term prospective studies awaiting disease-free survival and overall survival data. Currently our group is investigating the use of the assay to detect receptor-positive exosomes, which requires the development of a new algorithm using alternate normalizing genes for data normalization. The use of liquid biopsies and robust gene expression assays will allow high throughput multiplex assays adapted for patient management during 
treatment, and provide a means to follow treatment efficacy, potential relapse due to resistance to therapy, and the metastatic capacity of the tumor.

This method also has a wide range of possible applications in the diagnosis of tumors and is adapted to the current diagnostic workflow. The main advantages of this method in the diagnostic field include: (1) implementation of high throughput assays, (2) excluding subjectivity and equivocal results originating from image-based measurements, (3) accurate detection of multiple targets simultaneously, which enhance accuracy and minimize the use of precious patient samples, and (4) no requirement for highly specialized facilities and human resources. The optimized sampling process, together with the low input of material required for the bead-based multiplex assay, allows further investigation of tumor heterogeneity; by using laser microdissection to accurately separate multiple foci of malignant tissue from the same patient section, it is possible to compare multiple gene expression between them as well as with matched normal tissue (Figure 4). Low material input is vital for diagnostic application on tumor biopsies that provide limited tumor tissue. The capacity of the assay to measure gene expression from degraded RNA samples allows easy transportation of samples for analysis within an institution or to servicing laboratories. In addition, whole section analysis was also possible using H\&E stained material (Figure 1).

For the success of this protocol, it is imperative to: (1) ensure proper sampling of the tumor site/s that are lysed for the assay and (2) develop well optimized and validated data normalization algorithms, for each gene expression panel and/or individual prognostic or predictive biomarkers. The former depends on the technical experience of the technician/scientist performing the sampling. It is recommended to take an additional core and prepare a tissue microarray (TMA) in the same format of the multiplex magnetic bead assay (96-well format). This will provide an archive of tumor sites as a replica of samples used for the RNA-based assay. TMAs can also be assessed with other techniques for followup research or validation of results. The development of normalization algorithms is dependent on the material being investigated and the normalizing genes selected for normalization. Different panels of normalizing genes are selected based on the level and variability of expression in the sample analyzed and this varies between cancer tissues from different origins, exosomes from plasma, or circulating tumor cells. Validation of the assay includes sample processing since various preparations will also result in different normalization algorithms.

To summarize, the use of bDNA technology in combination with magnetic bead technology and the selection of the proper panel of target genes, will provide the added advantage of measuring gene expression directly in tissue lysates derived from small amounts of patient material, including microdissected material, exosomes, and circulating tumor cells. In addition to detection of tumor heterogeneity, the proper use of panels has the potential to detect tumor derived exosomes for early diagnostics and early detection of relapses. Since there is no need for a nucleic acid amplification step, the signal amplification using the bDNA technology, combined with the bead-based multiplex, measures multiple gene expression in clinically-annotated archival material and provide a resource for biomarker validation.

\section{Disclosures}

The Invitrogen ${ }^{\mathrm{TM}}$ QuantiGene ${ }^{\mathrm{TM}}$ Plex Assay is proprietary of Thermo Fisher Scientific. Production and Open Access publication of this videoarticle was sponsored by Luminex Corporation

\section{Acknowledgements}

The work was supported by (1) a Breast Cancer Project Scholarship (2014-2016) funded by the Action for Breast Cancer Foundation and ALIVE 2013 through the Research, Innovation \& Development Trust (RIDT) of the University of Malta, (2) the Faculty of Medicine \& Surgery, University of Malta and (3) Project ACT financed by the Malta Council for Science \& Technology through FUSION: The R\&I Technology Development Programme 2016. The publication of this manuscript is supported through the Jove-Luminex grant.

\section{References}

1. Abrahamsen, H. N., Steiniche, T., Nexo, E., Hamilton-Dutoit, S. J., \& Sorensen, B. S. Towards quantitative mRNA analysis in paraffinembedded tissues using real-time reverse transcriptase-polymerase chain reaction: a methodological study on lymph nodes from melanoma patients. J Mol Diagn. 5 (1), 34-41 (2003).

2. Macabeo-Ong, M. et al. Effect of duration of fixation on quantitative reverse transcription polymerase chain reaction analyses. Mod Pathol. 15 (9), 979-987 (2002).

3. Yang, W. et al. Direct quantification of gene expression in homogenates of formalin-fixed, paraffin-embedded tissues. Biotechniques. $\mathbf{4 0}$ (4), 481-486 (2006).

4. Flagella, M. et al. A multiplex branched DNA assay for parallel quantitative gene expression profiling. Anal Biochem. 352 (1), 50-60 (2006).

5. Goldhirsch, A. et al. Strategies for subtypes--dealing with the diversity of breast cancer: highlights of the St. Gallen International Expert Consensus on the Primary Therapy of Early Breast Cancer 2011. Ann Oncol. 22 (8), 1736-1747 (2011).

6. Schnitt, S. J. Classification and prognosis of invasive breast cancer: from morphology to molecular taxonomy. Mod Pathol. 23 Suppl 2 S60-64 (2010).

7. Bae, Y. K. et al. Fibronectin expression in carcinoma cells correlates with tumor aggressiveness and poor clinical outcome in patients with invasive breast cancer. Hum Pathol. 44 (10), 2028-2037 (2013).

8. Park, J., \& Schwarzbauer, J. E. Mammary epithelial cell interactions with fibronectin stimulate epithelial-mesenchymal transition. Oncogene. 33 (13), 1649-1657 (2014).

9. Zhou, Z. et al. MRI detection of breast cancer micrometastases with a fibronectin-targeting contrast agent. Nat Commun. 6 7984 (2015).

10. Antonyak, M. A. et al. Cancer cell-derived microvesicles induce transformation by transferring tissue transglutaminase and fibronectin to recipient cells. Proc Natl Acad Sci U S A. 108 (12), 4852-4857 (2011).

11. Moon, P. G. et al. Fibronectin on circulating extracellular vesicles as a liquid biopsy to detect breast cancer. Oncotarget. 7 (26), $40189-40199$ (2016).

12. Parker, J. S. et al. Supervised risk predictor of breast cancer based on intrinsic subtypes. J Clin Oncol. 27 (2009). 
13. Nielsen, T. et al. Analytical validation of the PAM50-based Prosigna Breast Cancer Prognostic Gene Signature Assay and nCounter Analysis System using formalin-fixed paraffin-embedded breast tumor specimens. BMC Cancer. 14 177-177 (2014).

14. Fischer, A. H., Jacobson, K. A., Rose, J., \& Zeller, R. Hematoxylin and Eosin Staining of Tissue and Cell Sections. Cold Spring Harbor Protocols. 2008 (5) (2008).

15. Molecular Machines and Industries. H\&E Staining Kit Plus for Laser Microdissection and more, Prod. No 70302. (2018).

16. Lin, F., \& Prichard, J. Handbook of Practical Immunohistochemistry: Frequently asked questions. Springer (2011).

17. Grech, G. et al. Molecular Classification of Breast Cancer Patients Using Formalin-fixed Paraffin-embedded Derived RNA Samples. Journal of Molecular Biomarkers \& Diagnosis. 7 (S8) (2016). 